\title{
COMPARATIVE ANALYSIS OF NEURO- FUZZY AND SIMPLEX OPTIMIZATION MODEL FOR CONGESTION CONTROL IN ATM NETWORK.
}

\author{
${ }^{1}$ Sochima Vincent Egoigwe, ${ }^{2}$ Stephen Sunday Okika, ${ }^{3}$ Timothy Oluwaseun Araoye, ${ }^{4}$ Chukwudozie Chukwudi \\ Michael and ${ }^{5}$ Nwobi Chukwudumebi Gibson \\ ${ }^{1,3}$ Department of Mechatronics Engineering, University of Nigeria, Nsukka, Nigeria. \\ ${ }^{2}$ Department of Electrical and Electronics Engineering, Enugu State University of Science and Technology, \\ Enugu State, Nigeria. \\ ${ }^{4,5}$ Department of Electronics Engineering, University of Nigeria, Nsukka, Nigeria. \\ Corresponding Author: Sochima Vincent Egoigwe
}

\begin{abstract}
Congestion always occurred when the transmission rate increased the data handling capacity of the network. Congestion normally arises when the network resources are not managed efficiently. Therefore if the source delivers at a speed higher then service rate queue, the queue size will be higher. Also if the queue size is finite, then the packet will observed delay. MATLAB Software was used to carry out simulations to develop Congestion control optimization Scheme for ATM Network with the aims to reducing the congestion of Enugu ATM Network. The results of the research reveal the minimization of congestion application model for Enugu ATM using optimization and Neuro-fuzzy. The result shows that congestion control model with Optimization and Neuro-fuzzy were 0.00003153 and 0.00002098 respectively. The ATM Congestion was reduced by 0.0000105, which is $18.2 \%$ decrease after Neuro-fuzzy controller was used. The results show the application of Neuro-fuzzy model which can use to control and minimized the ATM Congestion of Enugu ATM Network. The result shows that when Neuro-fuzzy is applied the congestion and the packet queue length in the buffer will be minimized.
\end{abstract}

Key words: Congestion, MATLAB, Optimization, Neuro-fuzzy, ATM

DOI: $10.7176 / \mathrm{CTI} / 10-05$

Publication date:July $31^{\text {st }} 2020$

\section{Introduction}

Congestion normally occurred when the transmission Source rate exceeds the data capacity of the network. The degradation in performance is observed. Congestion is said to be occurred if network resources are not efficiently managed. If the source delivers at a rate higher than service rate of queue, then the size of queue will grow. If the size of queue is finite, then packets will experience delay \& losses will occur. End users experience delay in delivery of packets, even loss of packets also occurred [3]. Networks that try to send more data than network capacity, lead to data loss and excessive delays in packet delivery [2]. Due to unstable fluctuations and burstiness of traffic flow in ATM networks, congestion occurs frequently [4]. There is choice that show the congestion and where can influence a choice of a control approach with respect to location [3]. Congestion control is a process in which networks adjusts the amount of input data, so that the Quality of Service (QoS) is not degraded and simultaneously try to utilize the network resources efficiently [2].The ATM technique provides an attractive solution to the problem of integrating different types of services, with widely different bitrates, through common interface and switching fabrics. It is a compromise between packet switching and circuit switching techniques. The traffic control set functions must be provided by the ATM network to ensure the QoS of each service and to produce a high network utilization. The wide range of service characteristics, such as bit rates, burstiness factors, cell delay constraints (latency), cell loss tolerance (accuracy), and priority combined with the need for adaptive, and sometimes real-time services makes the use of traditional control methods very difficult. Although ATM networks can support a wide variety of transmission rates and provide transmission efficiency by asynchronous multiplexing, a cell might be lost in ATM switches if cells are excessively fed into the networks. In order to avoid this situation, the terminals are required to declare their transmission rates as traffic parameters, e.g., peak cell rate (PCR) and sustainable cell rate (SCR), in advance of transmission. According to these declarations of transmission rates, ATM switches judge whether the required QoS can be achieved. if the QoS can be met without deteriorating those of the existing calls, then the call is admitted, otherwise it is rejected. This traffic control function for an ATM system, called connection admission control (CAC), decides whether to accept or reject a call based upon availability of capacity required to support its QoS. Thus an estimate of the 
QoS is required based on monitoring traffic patterns and buffer status, which is important in determining the cell loss probability, cell delay and delay variations.

The factor like the traffic source descriptor, the intensity of current network congestion along of the incoming path call, and QoS requirements of the pre-existing and new calls is a stressful task for any mathematical model.

Traffic Management and congestion control are major issues in ATM networks [1,8,11,20,17,21]. Congestion arises once incoming traffic to specific link is quite outgoing link capability. When congestion downside arises, cells square measure discarded while not being processed. The design of congestion control mechanism is essential not only for regulating traffic to prevent congestion but also for providing efficient and fair bandwidth allocation $[6,19]$. Among numerous congestion management mechanisms, rate base mechanisms square measure tested economical than different mechanisms. Rate based congestion control schemes are end-to-end feedback mechanisms, and the cell transmission rate of each source end system is regulated according to congestion feedback information returned by the network $[3,8,15]$. In rate primarily based congestion management schemes, supply finish system sporadically sends a Resource Management (RM) cell for each thirty one information cells. The RM cell is used to carry control and flow information over the connection between source and destination end systems [20]. The destination finish system sends associate degree RM cell with associate degree indicator showing the standing of traffic back to the supply finish system.

However the development of economic Network management resources is always stressful. With traditional network management methods it is difficult to obtain a comprehensive view of the state of the network and simultaneously this cover important detail from the traffic. When communication network is direct, The Asynchronous Transfer Mode (ATM) generates a method that looks very promising from the perspective of traffic engineering. ATM is a modern technology enabling the integration of different traffic types with a single communication network. ATM Networks is developed to carry voice, video and data traffics with high s peed links using short fixed size cells of 48 bytes of payload and 5 bytes of header. Therefore there are Various service classes which has been defined in ATM for traffic supports with different quality of service (QoS) requirements. These classes required constant bit rate (CBR), real-time variable rate (rt-VBR), non-real time variable bit rate (nrt-VBR), available bit rate (ABR) and unspecified bit rate (UBR). Of these, Available Bit Rate (ABR) service has been applied in order to support highly busty traffic data applications [11,27].

\section{ATM Network Management}

ATM network management is always difficult due to the various kind of traffic expected on ATM networks; hence, the standard of the Quality of Service (QoS) is much more challenging. In order to meet their strict QoS requirements, CBR and VBR sources are guaranteed bandwidth when they are admitted into the network. UBR traffic is simply minimally supported by the network. The functions of CAC and traffic isolation mechanisms are to manage enough bandwidth for CBR and VBR services and to control ATM network. ABR congestion control aims to maintain maximum ABR by adjusting each ABR source rate based on the total traffic status of the network. ATM ABR supports these 3 methods of act congestion performance from ATM switches and destination endsystems back to a supply device. These strategies names are binary, relative rate and explicit rate.

The flow management mechanism supply router will transmit cell rate information written by the intermediate ATM NEs within the payload of the RM cell. The RM cell can now control traffic of the ATM congestion through ABR. Most importantly, the flow-rate-control method is very important as a router places in the current cell rate (CCR) field[12,13].

\section{Related Works}

Researcher [28] realized the CAC via a probabilistic burstiness-curve, in which each session connection is defined by the buffer space and transmission bandwidth. [32] presented a uniform CAC scheme based on a Chernoff bound method that uses a simple novel traffic model requiring only a few parameters. Evans and Everitt [17] focused on the newly-developed CDMA cellular networks and proposed an effective bandwidth-based CAC method. The decision-making nature of CAC has attracted many re- searchers to apply FLSs and neural networks to it. Fuzzy logic systems (FLSs) are program to represent and numerically manipulate linguistic rules in an exceedingly natural approach and for his ability to handle issues that standard management theory cannot approach successfully because the latter relies on a valid and accurate model which does not always exist, and, FLSs have also been extensively used in system modeling.[24]. [2], [9] proposed a power-spectrum based neural fuzzy CAC for ATM networks. They constructed a decision hyper plane of the CAC according to the parameters of the power spectrum. They devised rules which used the following type-1 fuzzy sets in antecedent's sets: light load, medium load, and heavy load and the following type 1 fuzzy sets in consequent sets: straightly reject, weakly reject, weakly accept, and straightly accept. All of those rules are supported the information from one expert; however, words can mean different things to different people. 
[21] studied the possibility distribution of cell loss as a function of the number of calls per class, by a fuzzy inference scheme based on the observed data of cell ratio (CLR), and obtained the upper bound of CLR. They applied fuzzy illation to estimate the chance distribution of CLR, that was then a basis for admission management selections. [14] also proposed a CAC scheme using a type-1 FLS to estimate the level of traffic burstiness; they estimated hurts parameters and used them for CAC in an adaptive environment.

[15] proposed use of fuzzy logic prediction on connection Admission Control (CAC) and congestion control in high speed networks. Traffic predictions had been demonstrated with having the ability to improve system efficiency and QoS. Firstly Fuzzy Logic predictor was applied to congestion control in which ABR queue was estimated one round trip time in advance. It was shown that fuzzy logic scheme decreased the convergence time \& over all buffer needs as compared to traditional schemes. Secondly, they modelled traffic characteristic utilizing on-line fuzzy-logic predictor on CAC. By using simulation it was shown that fuzzy logic prediction enhanced the efficiency of both conventional as well as fuzzy based CAC. [2] described rate- feedback data flow control problem in one source single bottleneck communication system and proposed a fuzzy logic based controller that ensures stability performance. Main advantage of proposed solution was its simplicity as compared to other methods. It was shown that proposed controller is robust to uncertainty caused by communication channel delay. It was also shown that proposed controller stabilizes the system and also ensures to asymptotically regulate queue length to a desired steady state value [29]. [16] described existing transport layer protocols which performed well for most problems, but still offers some problems. As Transmission Control Protocol (TCP) tracks the current state of network situation \& can act appreciably to sign of congestion. TCP serves data-oriented applications very well but can lead to low quality for streaming media applications such as real-time video. The other protocol that was discussed is UDP, which is not friendly to TCP flows but ensures good provision for streaming media application and can cause congestion collapse problem. Author proposed a new protocol that utilizes Fuzzy Logic Controller (FLC) which by using network information such as available bandwidth, packet loss rate and round trip time generated the send rate to meet the requested rate in smooth way without wasting network resources. Author showed using simulation that controller reduces the chances of congestion collapse from undelivered packets, reacted to changes in network smoother than AIMD scheme utilized in TCP, provides incentive to application to use congestion control by utilizing network better than TCP and remains friendly to TCP flows [30][31].

\section{Fuzzy modeling}

The Application require mathematical model which corresponds to the actual inputs to outputs, where the aims is to approximate a function $y=f(x)$ with input $x=\left(x_{1}, x_{2}---x_{n}\right)$.

If $x_{1}$ is $C_{1} j$ AND $x_{2}$ is $c_{2 j}$

So, $x_{n}$ is $c_{n j}[19]$

THEN: yj is Dj, where Cij is a membership function of the input xi and cj with ouput Dj. The defuzzifier is:

$y=\frac{\int_{\alpha}^{\alpha} \mu(y) \cdot y d y}{\int_{\alpha} \mu(y) \cdot d y}$ where $\mu(y)=\cup\left[\min \left(\prod_{i=1}^{n} \mu_{i j(x)}\right)\right], V_{j}(y)$

$\mathrm{V}_{\mathrm{i}}(\mathrm{y})$ represent the degree of membership of the output $\mathrm{y}_{\mathrm{j}}$ to $\mathrm{C}_{\mathrm{j}}$

$\mu_{i j}(x)$ represent degree of membership of input $\mathrm{xi}$ to the fuzzy set $\mathrm{c}_{\mathrm{ij}}$.

\section{Neurofuzzy Models}

Neurofuzzy models [28] have been applied successfully in non-linear model based techniques [22]. This provide optimization model for the parameter in the fuzzy system which gives the best fits[20]. These models may be formulated as an Adaptive Neuro-Fuzzy Inference System (ANFIS) [18]. ANFIS is presented as an example with $\mathrm{N}$ input variables, one output variable and five layers. The first layer is composed of membership functions $A_{u i j}$, defined by the membership degree[24]

$\mu_{\text {Auij }}: u_{i} \in \mathrm{R} \rightarrow \mu_{A u i j}\left(u_{i}\right) \in \mathrm{R}$ 


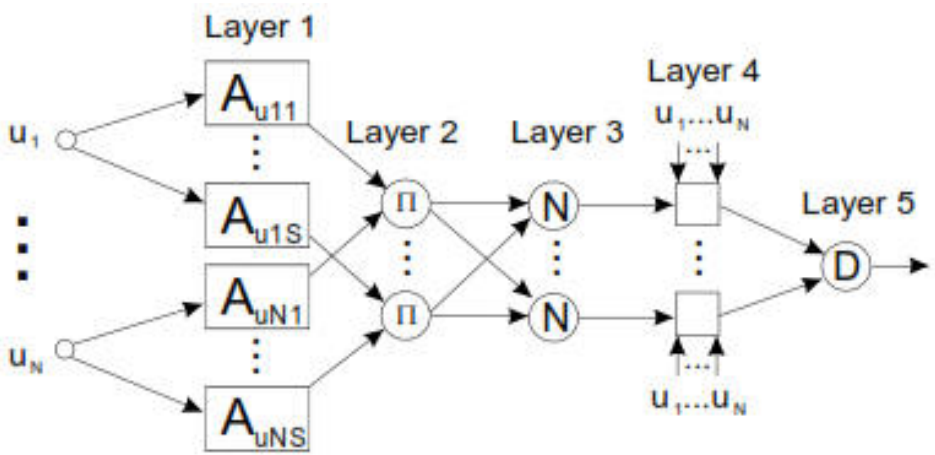

Figure1: Fuzzy Neural Network [18]

The output of each node $i$ is $\mu_{A u i j}\left(u_{i}\right)$, the membership degree of $u_{i}$. For the definition of these membership functions, some standard types are used. In this work, gaussian membership functions are used. The second layer has nodes labelled with $\Pi$ which implement fuzzy inference machine. For example, if logical operation $A N D$ is carried out by multiplication, the output of each node $j$ of this layer is:

$$
\begin{gathered}
\omega_{j}=\mu_{A \boldsymbol{u} 1 j}\left(u_{1}\right) \cdot \mu_{A u 2 j}\left(u_{2}\right) \cdots \cdot \mu_{A \boldsymbol{u} N_{j}}\left(u_{N}\right) \\
W_{i}^{-}=\frac{w_{i}}{\sum_{i=1}^{N} w_{i}}
\end{gathered}
$$

The fourth layer has adaptive nodes:

$$
\stackrel{-}{W}_{i} f_{i}=w_{i}\left(p l_{i} u_{i}+--+p N_{i} u N+r_{i}\right)
$$

Finally, the fifth layer is the defuzzyfication node: for TS systems, the output will be

$$
\sum_{i=1}^{N} \bar{w}_{i} f_{i}=\frac{\sum_{i=1}^{N} w_{i} f_{i}}{\sum_{i=1}^{N} w_{i}}
$$

Fuzzy Neural Networks (FNN) combine the capability of uncertainty handling in information with learning skills [18], [6]. Recurrent Fuzzy Neural Networks (RFNN) have demonstrated to be better at getting all the dynamics of nonlinear systems. They are systems which have the same advantages as recurrent neural networks [5],[7]. RFNN are also named Fuzzy Dynamical Systems and extend the application domain of FNN to temporal problems. Feedback allows the capture of dynamics and change. In the neurofuzzy model proposed by Takagi-Sugeno(TS), the structure of antecedent describes fuzzy regions in the input space, and the one of consequent presents non-fuzzy functions of the model inputs. If recurrent functions with NARMAX structure (Non-linear Auto Regressive Moving Average with eXogenous input $)$ of the kind:

$$
\hat{y}(k+1)=f(y(k), \ldots, y(k-m), u(k), \ldots, u(k-n))
$$

are used, being $u, y$ for each rule the inputs and outputs of the system respectively, the system may be described by the following way:

IF $x_{1}(k)$ is $F_{1 j}, \ldots$, and $x_{n}(k)$ is $F_{n j}$,

THEN: $y_{\boldsymbol{j}}(k)=a_{\boldsymbol{j}}\left(z^{-1}\right) y(k-1)+b_{\boldsymbol{j}}\left(z^{-1}\right) u(k-d)+c_{\boldsymbol{j}}$

With $a_{j}\left(z^{-1}\right)=a_{1 j}+a_{2 j} z^{-1}+\ldots+a_{n} j^{-(n y-1)}$ and $b_{j}\left(z^{-1}\right)=b_{0 j}+b_{1 j} z^{-1}+b_{2 j} z^{-2}+$ $\ldots+b_{n} j^{z}{ }^{-n_{u}}$ and where $X(k)=\left[x_{1}(k) x_{2}(k) \ldots x_{n}(k)\right]^{T}$ is the input vector of the neurofuzzy system in the instant $k, F_{i j}$ is the fuzzy set respective to $x_{i}(k)$ on the rule $j, y_{j}(k)$ is the output of the model respective to the operating region associated to the rule, $d$ is the dead time and $c_{j}$ is a constant term. If $\mu_{i j}(k)$ is the membership degree of $x_{j}(k)$ in the fuzzy set $F_{i j}$ and the number of implications or rules is $L$, the RFNN complete model is possibly described by: 


$$
y(k)=\sum_{j=1}^{L} w_{j}(k)\left[a_{j}\left(z^{-1}\right) y(k-1)+b_{j}\left(z^{-1}\right) u(k-d)\right]+\xi(k) .
$$

Where $w_{j}(k)=\frac{\mu_{j}(k)}{\sum_{j=1}^{L}-\bar{\mu}(k)} \quad \bar{\mu}_{j}(k)=\prod_{i=1}^{n} \mu_{i j}(k)$ and $\xi(k)=\sum_{j=1}^{L} w_{j}(k)$.

\section{Materials and Method}

This paper develops comparative analysis of Neuro-fuzzy and Optimization control for ATM Network Congestion. The control optimization was designed to minimized congestion occurs, and the packets accumulate in the buffer allocated for that link. The feedback mechanism for the input rate regulation is provided by means of control units emitted periodically by each source. The congestion control scheme for ATM Network was analyzed using MATLAB/SIMULINK Software.

\section{A. Mathematical Model of Congestion Control.}

These special units travel along the same path as data packets. However, unlike data packets, they are not stored in the queues at the intermediate nodes. Instead, once they appear at the node input link and the feedback information is incorporated, they are immediately transferred at the appropriate output port. As soon as control units reach the destination, they are turned back to be retrieved at the origin, and to be used for the transfer speed adjustment round trip time after they have been generated. The packet queue length in the buffer, which at time nT will be denoted as $\mathrm{z}(\mathrm{nT})$, and its demand value yd, are used to calculate the current amount of data $\mathrm{u}(\mathrm{nT})$ to be sent by the sources. The mth share of the total amount, $\mathrm{u}(\mathrm{kT}) / \mathrm{m}$, is recorded as the feedback information in every management unit passing through the node[25].

$$
Z(n T)=\sum_{p=1}^{m} \sum_{i=0}^{n-1} \frac{1}{m} u\left(i T-R T T_{P}\right)-\sum_{i=0}^{n-1} h(i T)=\sum_{j=1}^{n m} \frac{\beta_{j}}{m} \sum_{i=0}^{n-j-1} u(i T)-\sum_{i=0}^{n-1} h(i T)
$$

The Network can be described in a state space as

$$
\begin{aligned}
& x[(n+1) T]=A x(n T)+b u(n T)+o h(n T) \\
& Z(n T)=q^{T} x(n T)
\end{aligned}
$$

Where

$$
x(n T)=\left[x_{1}(n T) x_{2}(n T)---x_{n}(n T)\right]^{T}
$$

\section{B. Optimization Modeling}

The empirical or measured data collected from Enugu MTN Network was used to develop a Mathematical model for minimizing the congestion of the ATM Network using Linear Programing.

Maximize $Z=452 X_{1}+595 X_{2}+576 X_{3}+734 X_{4}$

Subject to

$452 \mathrm{X}_{1}+595 \mathrm{X}_{2}+576 \mathrm{X}_{3}+734 \mathrm{X}_{4} \leq 0.07681$

$452 \mathrm{X}_{1}+595 \mathrm{X}_{2}+576 \mathrm{X}_{3}+734 \mathrm{X}_{4} \leq 0.06695$

$452 \mathrm{X}_{1}+595 \mathrm{X}_{2}+576 \mathrm{X}_{3}+734 \mathrm{X}_{4} \leq 0.2111$

$452 X_{1}+595 X_{2}+576 X_{3}+734 X_{4} \leq 0.06027$

Where $X_{1}$ to $X_{4}$ is congestion for four days

$\mathrm{Z}$ is the total congestion experienced for the four days 


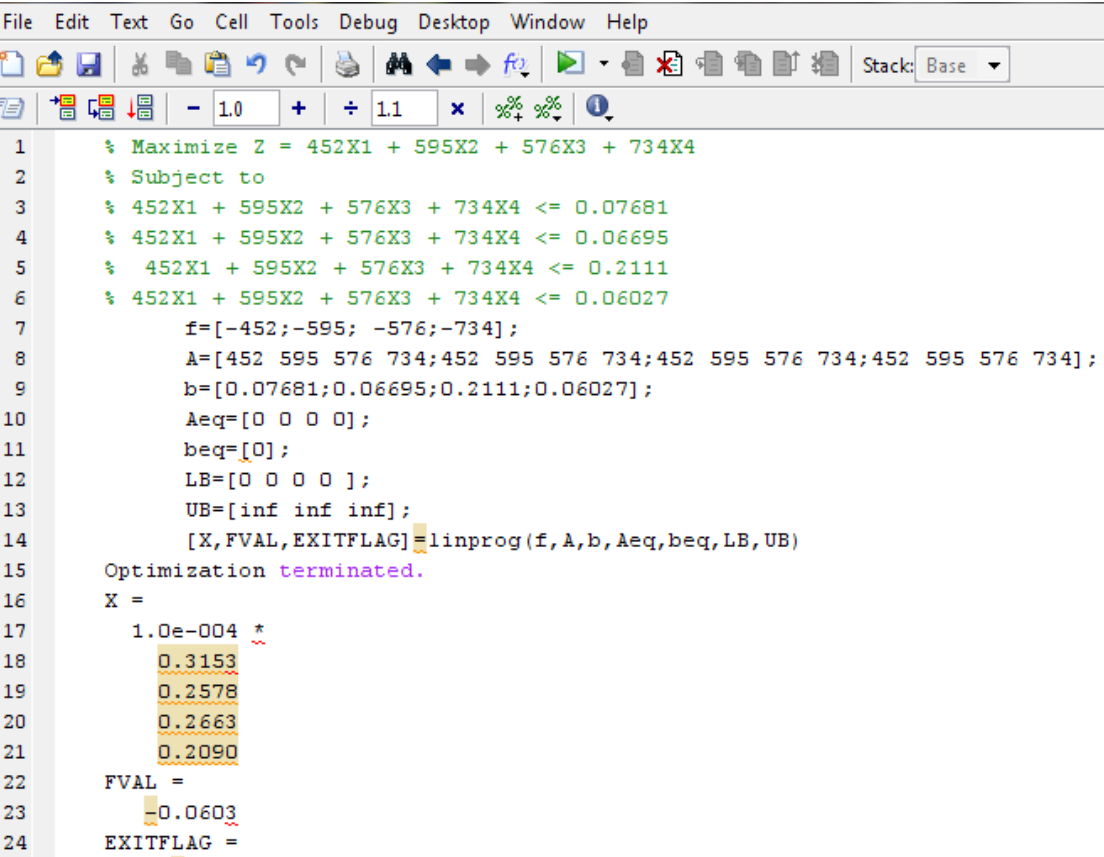

Fig 1 optimized the congestion from the characterized network.

Fig 1 shows optimized congestion from the characterized network for four days. The congestion result obtained in four days are $\mathrm{X} 1=0.00003153, \mathrm{X} 2=0.00002576, \mathrm{X} 3=0.00002663$ and $\mathrm{X} 4=0.00002080$.respectively. Table 1 Comparing reduction of congestion in optimized congestion control scheme for ATM network without and with neuro- fuzzy controller

\begin{tabular}{|l|l|l|}
\hline OPTIMIZED CONGESTION & $\begin{array}{l}\text { NEURO-FUZZY } \\
\text { CONGESTION }\end{array}$ & NUMBER OF USERS \\
\hline 0.00003153 & 0.00002098 & 1 \\
\hline 0.00002978 & 0.00001982 & 2 \\
\hline 0.00002663 & 0.00001772 & 3 \\
\hline 0.00002090 & 0.00001391 & 4 \\
\hline
\end{tabular}




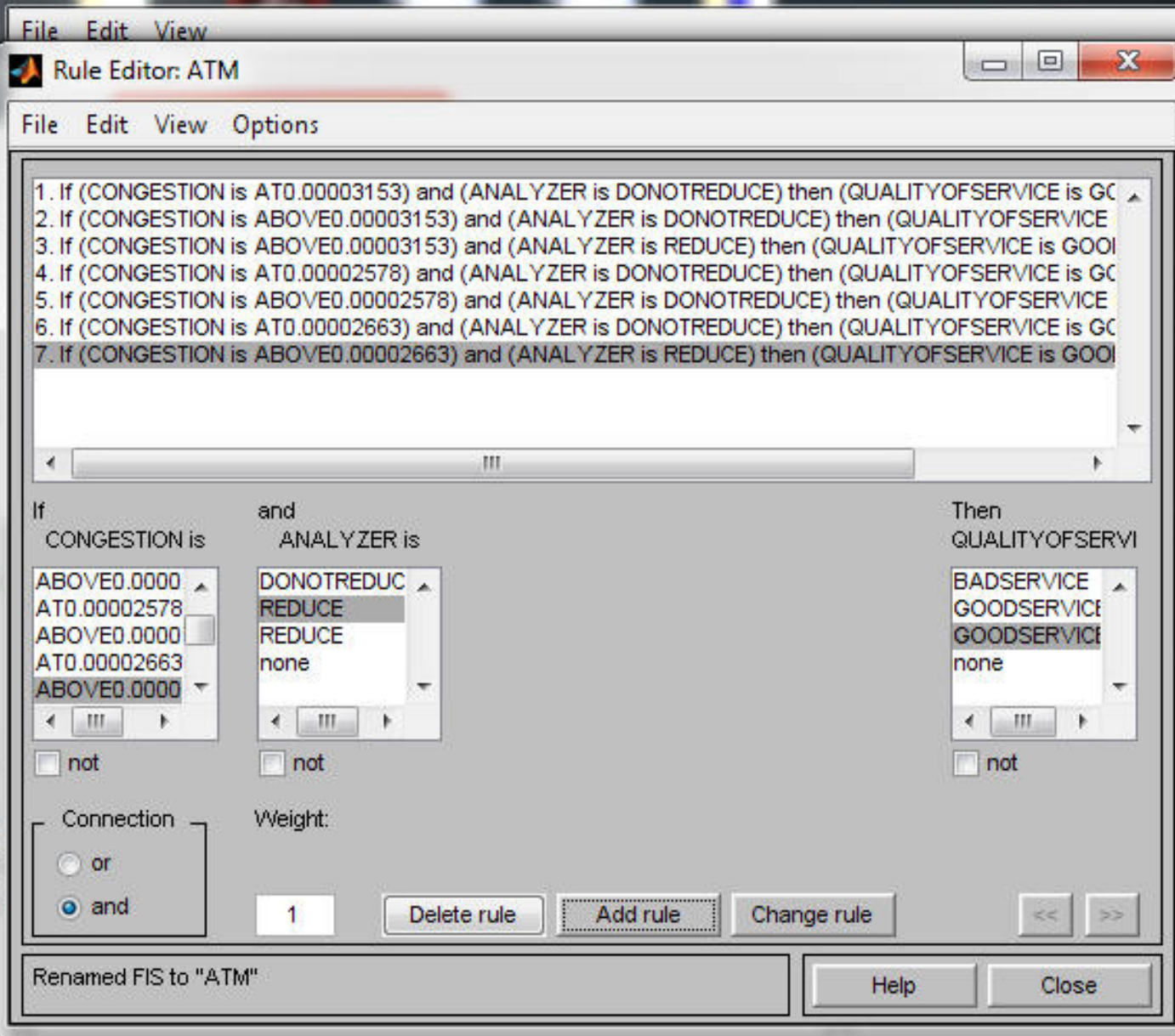

Fig 2 designed fuzzy rule to reduce congestion in ATM network using neuro- fuzzy 


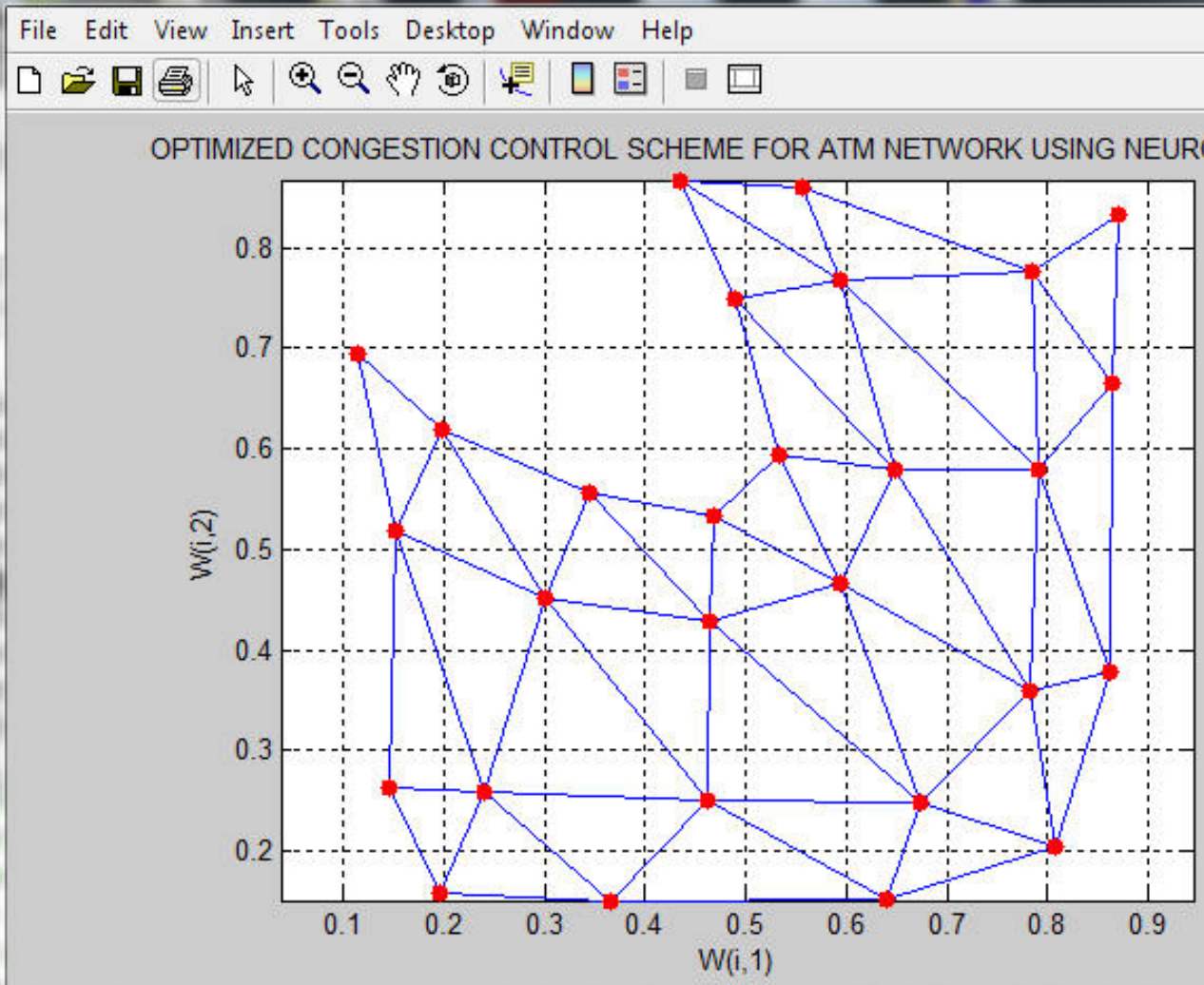

Fig 3 trained rules in ANN for optimized congestion control scheme for ATM network without using neurofuzzy

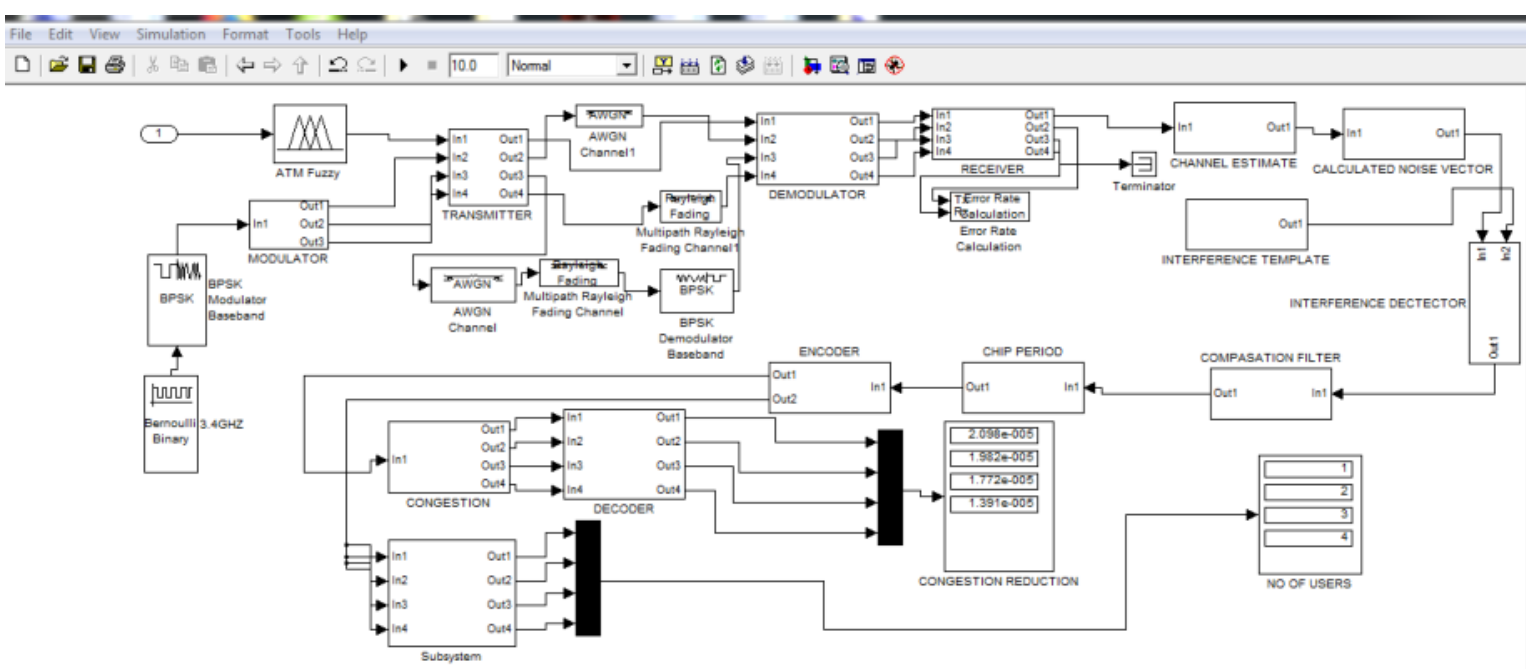

Fig 4 designed Simulink model for optimized congestion control scheme for ATM network using neuro fuzzy 


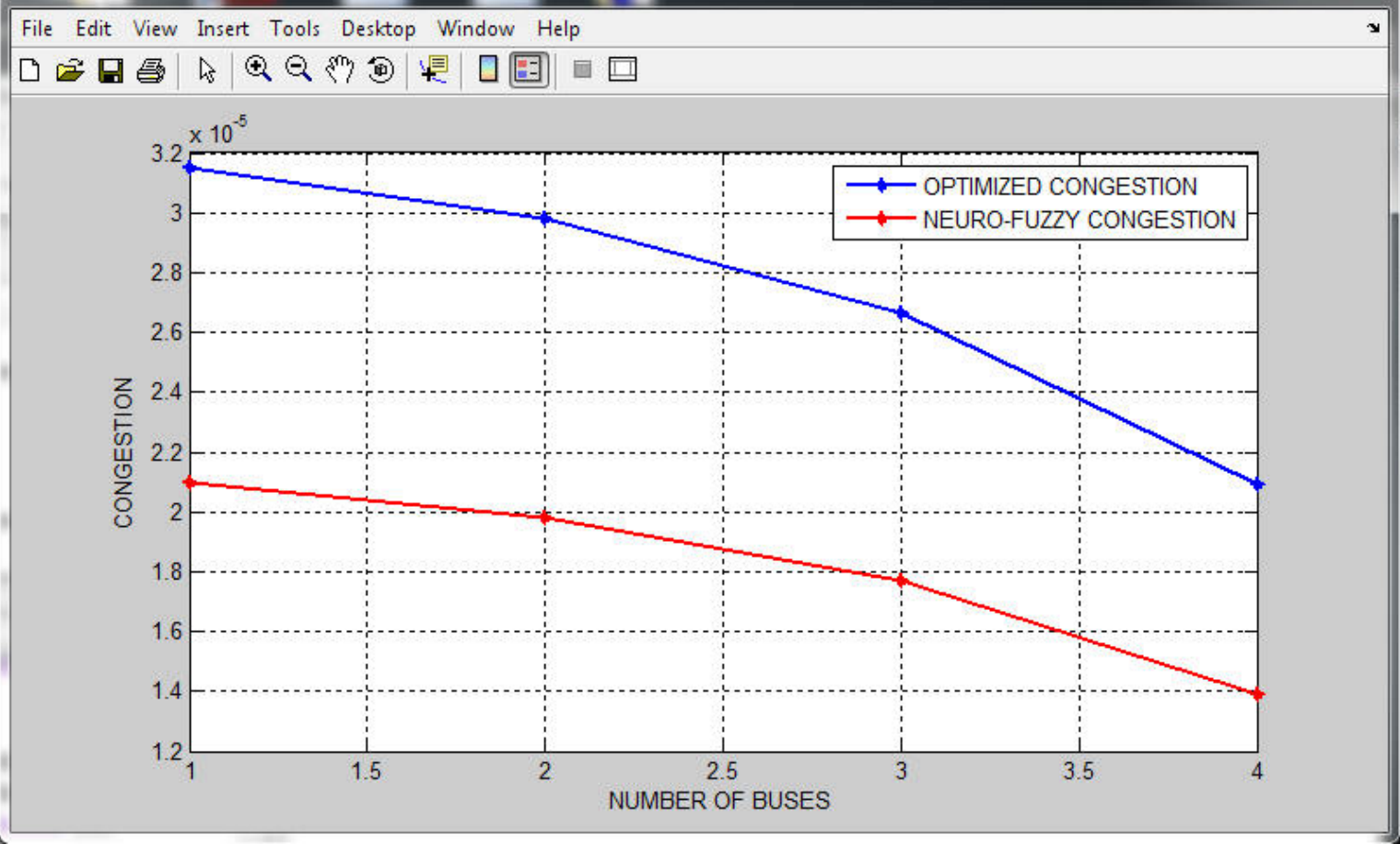

Fig 5 Comparing reduction of congestion in optimized congestion control scheme for ATM network without

and with neuro- fuzzy controller.

\section{Discussion of Result}

Fig 1 shows optimized congestion from the characterized network for four days. The congestion result obtained in four days are $\mathrm{X} 1=0.00003153, \mathrm{X} 2=0.00002576, \mathrm{X} 3=0.00002663$ and $\mathrm{X} 4=0.00002080$.respectively. This shows that congestion was reduced when optimized.

Fig 2 shows designed fuzzy rule to reduce congestion in ATM network using neuro-fuzzy. This shows the rules that that will reduce the optimized congestion in the ATM network.

Fig 3 shows trained rules in ANN for optimized congestion control scheme for ATM network without using neurofuzzy. This trained rules helps to maintain stable and free network.

Fig 4 shows designed simulink model for optimized congestion control scheme for ATM network. When the optimization model was used, the congestions observed in four days are $0.00003153,0.00002978,0.00002663$, 0.00002090 and $0.00002098,0.00001982,0.00001772,0.00001391$ respectively when Neuro-fuzzy controller was used. This result obtained is better than the field congestion computed.

Fig 5 shows designed Simulink model for optimized congestion control scheme for ATM network using neurofuzzy. This shows the simulated congestion result when neuro -fuzzy was incorporated in the system. therefore there is reduction in congestion when neuro- fuzzy is imbibed in the system than when optimization only is used.

\section{Conclusion}

ATM Congestion always occurred when the transmission rate increased the data handling capacity of the network. Congestion normally arises when the network resources are not managed efficiently. Therefore if the source deliver at a speed higher then service rate queue, the queue size will be higher. Also if the queue size is finite, then the packet will observed delay. MATLAB Software was used to carry out simulations to develop Congestion control optimization Scheme for ATM Network with the aims to reducing the congestion of Enugu ATM Network. The results of the research reveal the minimization of congestion application model for Enugu ATM using optimization and Neuro-fuzzy. The result showed that congestion optimization control model with Optimization and Neuro-fuzzy were 0.00003153 and 0.00002098 respectively. The ATM Congestion was reduced by 0.0000105 , which is $18.2 \%$ decrease after Neuro-fuzzy controller was 
used. The results show the application of Neuro-fuzzy model which can used to control and minimized the ATM Congestion. The result show that when Neuro-fuzzy is applied the congestion will be minimized packet queue length in the buffer is control then ATM Congestion will be minimal.

\section{Reference}

[1]A Hac, H.Lin, "Congestion Control for ABR traffic in an ATM network (1999)", International Journal of Network Management, Vol. 9, pp. 249-264.

[2]Ahmad, Khalid. Sourcebook of ATM and IP Internetworking. New York : IEEE Press,2002. pp 78-88

[3]Andreas Pitisillides and Ahmet Sekerciouglu "Fuzzy Logic based Congestion Control" http://Citeseerx.ist.psu.edu/viewdoc/ summery?doi=10.1.1.69.4324.

[4]Andreas Pitsillides, Y. Ahmet Sekercioglu and Gopalakrishnan Ramamurthy (1997) "Effective Control of Traffic Flow in ATM Networks Using Fuzzy Explicit Rate Marking (FERM)" IEEE Journal on selected areas in Communications, Vol 15, NO 2, pp.209-225.

[5]Anna Hac, Yingjun Ma, (1998) "A Rate based Congestion Control Scheme for ABR service in ATM networks", International Journal of Network Management, Vol.8, pp. 292-317.

[6]Bay Pavlick "A Fuzzy Logic based controller to provide end-to-end Congestion Control in Streaming Media Application (2005)" Master of Science Dissertation, University of South Florida, United States of America, pp.1-57.

[7]C.-H. Lee, C.-C. Teng (2000)“Identification and Control of Dynamic Systems Using Recurrent Fuzzy Neural Networks" IEEE Transactions On Fuzzy Systems 8(4), pp 349-366

[8]C.-J. Chang, C.-H. Lin, D.-S. Guan, and R.-G. Cheng, (1997)“A power-spec- trum based neural fuzzy connection admission mechanism for ATM net- works,” in IEEE Proc. ICC, Monterey, CA, pp. 1709-1713.

[9]Ching-Fong Su, Gustavo Do Vacianna, Jean Warland, (2000)“Explicit Rate Flow Control for ABR Services in ATM Networks", IEEE/ACM Transactions on Networking, Vol. 8, Issue 3, pp. 350-361.

[10]D Gaiti, G. Pujolle, "Performance management issues in ATM networks (1996): traffic and congestion control", IEEE/ACM transactions on networking, Vol. 4, No.2, pp.249-257.

[11]Design of a power-spectrum-based ATM connection admission controller for multimedia communications," IEEE Trans. Ind. Electron., vol. 45, pp. 52-59, Feb. 1998.

[12]E Al-Hammadi and M M Shasavari, (2000) "Engineering ATM networks for congestion avoidance", Mobile Networks and Application, Vol. 5, pp.157 - 163.

[13]H Ohsaki, M Murata, H Suzuki et al, (1995)“Rate based Congestion Control for ATM networks”, ACM SIGCOMM, Computer Communication Review, Vol.25, Issue 2, pp. 60-72.

[14]H. R. Mehrvar and T. Le-Ngoc, (1997)“Fuzzy logic in estimation of traffic burstiness for admission control in broadband networks," in Proc. IEEE ICC, Monterey, CA, pp. 1090-1094.

[15]Hiam Hiok Lim and Bin Qiu (2201)“Fuzzy Logic Control in Broadband Communication Networks" Proceedings of IEEE International Fuzzy Systems Conference, pp.99-102.

[16]Held G. (1999), Asynchronous Transfer Mode. http://technet.microsoft.com,10 May 2010.

[17]J. S. Evans and D. Everitt, (1999)"Effective bandwidth-based admission control for multiservice CDMA cellular networks,” IEEE Trans. Veh. Technol., vol. 48, pp. 36-46.

[18] J.S.R. Jang, C.T. Sun, E. Mizutani. (1997) "Neuro-fuzzy and Soft Computing" Prentice-Hall

[19]J. Zrida, A. Benzaouia, F. Mesquine and S. El Faiz "Rate-Based Flow Fuzzy Controller for Communication Systems" Proceedings of $1^{\text {st }}$ African Control Conference, Cape Town, South Africa, 2003, pp. 120-123.

[20]K Siu, H Tzeng, "Intelligent Congestion Control for ABR service in ATM networks",ACM SIGCOMM Computer Communication Review, Vol. 25, Issue 2, pp. 81-106, 1994.

[21]K. Uehara and K. Hirota, "Fuzzy connection admission control for ATM networks based on possibility distribution of cell loss ratio," IEEE J. Select. Areas Commun., vol. 15, pp. 179-190, Feb. 1997.

[22]K. Kiriakidis, "Fuzzy Model-Based Control of Complex Plants" IEEE Transactions On Fuzzy Systems 6(4), pp. 517-529 (1998).

[23]Lalita A. Kulakarni, San-qi Li, "Performance Analysis of a Rate-based Feedback Control Scheme", IEEE/ACM Transactions on Networking, Vol. 6, No. 6, Decemeber 1998.

[24]M. Delago and A. Gonzalez, “An inductive learning procedure to identify fuzzy systems," Fuzzy Sets Syst., vol. 55, no. 2, pp. 121-133, 1993.

[25] Przemyslaw Ignaciuk. "congestion control in connection-oriented data transmission Network." Technical University of Lodz. 2009

[26]R.S.Deshpande, Dr. P.D. Vyavahare, "Recent Advances and a survey of congestion control mechanisms in ATM networks", IE(I) Journal, Vol.88, pp. 47-54, , 2007. 
[27]Rama Krishnan, "Rate based Control Schemes for ABR Traffic - Design Principles and performance comparison", Global Communication Conference Globecom96, pp.1231-1235, 1996.

[28]S. Chong and S.-Q. Li, "Probabilistic burstiness-curve-based connection control for real-time multimedia services in ATM networks," IEEE J. Select. Areas Commun., vol. 15, pp. 1072-1086, Aug. 1997.

[29]T. Takagi and M. Sugeno, "Fuzzy identification of systems and its applications to modelling and control" IEEE Trans. Syst., Man, Cybern.15, pp. 116-132 (1985)

[30]Thomos M.Chen, Steve S. Lin, David Wang, Vijay K.Samalam, Michael J. Procanik, and Dinyar Kavouspour, "Monitoring and control of ATM networks using special cells", IEEE network magazine, pp.28-38, Sept/oct 1996.

[31]W $\mathrm{Li}, \mathrm{Z}$ Che, $\mathrm{Y} \mathrm{Li}$, "Research on the congestion control of Broadband Integrated Service Digital Network based on ATM", Proceedings of the fifth international conference on Machine Learning and Cybernetics, Daliaan, pp 2510-2512, 2006.

[32]Z.-L. Zhang, J. Kurose, J. D. Salehi, and D. Towsley, "Smoothing, statistical multiplexing, and call admission control for stored video," IEEE J. Select. Areas Commun., vol. 15, pp. 1148-1166, Aug. 1997. 